\title{
THE SOLUTION OF THE DIFFERENTIAL EQUATION $\left(a^{2} \partial^{2} / \partial t^{2}-\Delta\right)\left(\partial^{2} / \partial t^{2}-\Delta\right) u=f(x, y, z, t)$ \\ BY HADAMARD'S METHOD
}

\section{J. P. KORMES}

1. Introduction. Hadamard $[1]^{1}$ generalized and extended Riemann's [2] method of solution of the initial value problem to any hyperbolic linear differential equation of the second order in any number of variables. The essential part of Hadamard's work is his discovery of the significance of an elementary solution which is a function of the geodetic distance determined by the characteristic manifold of the differential equation. Given the differential equation $L[u]=f$ and the initial data on a manifold $C$, let $\Gamma=0$ be the equation of the characteristic manifold of the differential equation and $M[u]$ the adjoint expression of $L[u]$. The first step is the determination of an elementary solution $V$, that is, a solution of $M[V]=0$ which is singular on $\Gamma=0$. To obtain the solution of the initial value problem, Hadamard considers the domain bounded by the characteristic "conoid" through a point $P$, and the initial manifold $C=0$. Since the elementary solution is singular on the characteristic conoid, in order to apply Green's formula Hadamard uses an interior domain $G_{\epsilon, \delta}$ which approaches the original domain as $\epsilon \rightarrow 0$ and $\delta \rightarrow 0$. For a fixed $\delta$ each term of Green's formula has then the following form:

$$
B(\epsilon)=b+\left(1 / \epsilon^{(n-2) / 2}\right)\left(b_{0}+b_{1} \epsilon+\cdots+b_{(n-3) / 2} \epsilon^{(n-3) / 2}\right)+(\epsilon),
$$

where $(\epsilon)$ tends to zero as $\epsilon \rightarrow 0$ and the $b_{i}$ are independent of $\epsilon$. The term $b$, the "finite part" of the integral, has the property to remain invariant under all transformations of the parameter $\epsilon$. It follows from Green's formula that the sum of all such "finite parts" is equal to $z$ ero, which gives a relation for the function $u$. As we let the parameter $\delta$ approach zero we obtain the desired expression for $u(P)$. Hadamard, and independently Friedrichs [3], showed that in the case of an even number of variables each term of Green's formula will have an element containing $\log \epsilon$. The coefficients of $\log \epsilon$ have the property to remain invariant under all transformations of the parameter $\epsilon$ and it follows from Green's formula that the sum of all coefficients of $\log \epsilon$ is equal to zero, which gives the desired relation for the function $u$.

Received by the editors March 29, 1944.

${ }^{1}$ Numbers in brackets refer to the Bibliography at the end of the paper. 
The purpose of this paper is to show by a significant example that this method can be extended to problems of a more complex type.

I wish to express my appreciation to Professor R. Courant who suggested the subject and the method of attack, as well as to Dr. C. De Prima and Dr. B. Friedman for their generous assistance.

2. Elementary solution, Green's formula and domain of integration. We consider the initial value problem:

$$
\begin{aligned}
& L[u] \equiv\left(a^{2} \partial^{2} / \partial t^{2}-\Delta\right)\left(\partial^{2} / \partial t^{2}-\Delta\right) u=f(x, y, z, t) ; \quad a>1 ; \\
& u(x, y, z, 0)=u_{t}(x, y, z, 0)=u_{t t}(x, y, z, 0) \\
& =u_{t t t}(x, y, z, 0)=0 \text {, }
\end{aligned}
$$

where $\Delta$ is the Laplace operator: $\Delta=\partial^{2} / \partial x^{2}+\partial^{2} / \partial y^{2}+\partial^{2} / \partial z^{2}$, and the function $f(x, y, z, t)$ is assumed to have continuous derivatives up to the fourth order. The characteristic manifold is given by:

$$
\left[(\tau-t)^{2}-r^{2}\right] \cdot\left[(\tau-t)^{2} / a^{2}-r^{2}\right]=0,
$$

where $r^{2}=(\xi-x)^{2}+(\eta-y)^{2}+(\zeta-z)^{2}$. We introduce the functions $\Gamma$ and $\Gamma^{\prime}$ :

$$
\Gamma=(\tau-t)^{2}-r^{2} ; \quad \Gamma^{\prime}=(\tau-t)^{2} / a^{2}-r^{2} .
$$

The characteristic manifold consists of two conical sheets, the exteror: $\Gamma=0$, and the interior: $\Gamma^{\prime}=0$.

As the elementary solution $V$ of the self-adjoint equation $L[V]=0$ we take:

$$
V=-\frac{1}{2 r} \log \frac{(\tau-t)-r}{(\tau-t)+r}+\frac{a}{2 r} \log \frac{(\tau-t) / a-r}{(\tau-t) / a+r} .
$$

In order to obtain Green's formula for equation (2.1) we consider:

$$
\begin{array}{r}
Q[u, V]=a^{2} u_{t t} V_{t t}-a^{2} \sum_{i} u_{t t} V_{i i}-\sum_{i} V_{t t} u_{i i}+\sum_{i, k} u_{i i} V_{k h} \\
(i, k=x, y, z) .
\end{array}
$$

The integration of $Q[u, V]$ over a four-dimensional domain $G$ gives rise to two types of integrals:

(a) $\iiint \int_{G} u_{i i} V_{i i} d x d y d z d t$ and (b) $\iiint \int_{G} u_{i i} V_{k k} d x d y d z d t$
$(i, k=x, y, z, t)$.

Integrating by parts and collecting the results we obtain the following Green's formula: 


$$
\begin{aligned}
\iiint \int_{G}\{V L[u] & -u L[V]\} d g=\iiint_{S}\left\{V \frac{\partial N[u]}{\partial s_{2}}\right. \\
& \left.-u \frac{\partial M[V]}{\partial s_{1}}-N[u] \frac{\partial V}{\partial s_{2}}+M[V] \frac{\partial u}{\partial s_{1}}\right\} d o
\end{aligned}
$$

where:

$$
M=a^{2} \partial^{2} / \partial t^{2}-\Delta ; \quad N=\partial^{2} / \partial t^{2}-\Delta
$$

and where

$$
\partial / \partial s_{1}=t_{\nu} \partial / \partial t-x_{\nu} \partial / \partial x-y_{\nu} \partial / \partial y-z_{\nu} \partial / \partial z
$$

and

$$
\partial / \partial s_{2}=a^{2} t_{\nu} \partial / \partial t-x_{\nu} \partial / \partial x-y_{\nu} \partial / \partial y-z_{\nu} \partial / \partial z
$$

are the transversal differentiations, $x_{\nu}, y_{\nu}, z_{\nu}, t_{\nu}$ being the direction coefficients of the outward normal to $S$. If in $Q[u, V]$ we interchange $u$ and $V$ we obtain a second form of Green's formula:

$$
\begin{aligned}
& \iiint \int_{G}\{V L[u]-u L[V]\} d g=\iiint_{S}\left\{V \frac{\partial M[u]}{\partial s_{1}}\right. \\
&\left.-u \frac{\partial N[V]}{\partial s_{2}}-M[u] \frac{\partial V}{\partial s_{1}}+N[V] \frac{\partial u}{\partial s_{2}}\right\} d o .
\end{aligned}
$$

For the elementary solution $V(2.2)$ we find readily:

$$
M[V]=2\left(a^{2}-1\right)(\tau-t) / \Gamma^{2} ; N\lceil V\rceil=2\left(a^{2}-1\right)(\tau-t) / a^{2} \Gamma^{\prime 2} .
$$

We consider the domain $G$ bounded by the hyperplane $t=0$ and the exterior sheet $\Gamma=0$ through a point $P(\xi, \eta, \zeta, \tau)$. The domain $G$ is characterized by the inequalities: $\Gamma \geqq 0 ; 0 \leqq t \leqq \tau$. In accordance with Hadamard's method we truncate the domain $G$ by a plane $t=\tau-\delta$ and obtain the domain $G_{\delta}$ for which $\Gamma \geqq 0,0 \leqq t \leqq \tau-\delta, \delta>0$.

Since $\Gamma^{\prime}$ vanishes in the interior of $G_{\delta}$, the elementary solution has there a singularity. In order to enable us to apply Green's formula, we subdivide $G_{\delta}$ into the following domains:

(a) the conical ring $G_{\epsilon_{1} e_{2} \delta}^{1}$ :

$$
\begin{array}{cc}
0 \leqq t \leqq \tau-\delta ; & \\
\left(1-\epsilon_{1}\right)^{2}(\tau-t)^{2}-r^{2}>0 ; & 0<\epsilon_{1}<1-1 / a, \\
\left(1 / a+\epsilon_{2}\right)^{2}(\tau-t)^{2}-r^{2}<0 ; & 0<\epsilon_{2}<1-1 / a,
\end{array}
$$

(b) the truncated interior cone $G_{\epsilon_{8} 8}^{2}$ :

$$
\left(1-\epsilon_{3}\right)^{2}((\tau-t) / a)^{2}-r^{2}>0 ; \quad 0<\epsilon_{3}<1 .
$$


The ring domain is bounded by the volume $B_{e_{1} e_{2}}^{1}$, the lateral surfaces $L_{e_{1 \delta} \delta}^{1}$ and $L_{\epsilon_{2} \delta}^{2}$, and the volume $D_{\epsilon_{1} \varepsilon_{2} \delta}^{1}$. Similarly the domain $G_{e_{8} \delta}^{2}$ is bounded by the sphere $B_{\iota_{8}}^{2}$, the lateral surface $L_{\iota_{3}}^{2}$, and the sphere $D_{\mathbf{e}^{8} \mathrm{\delta}}^{2}$ (see diagram).

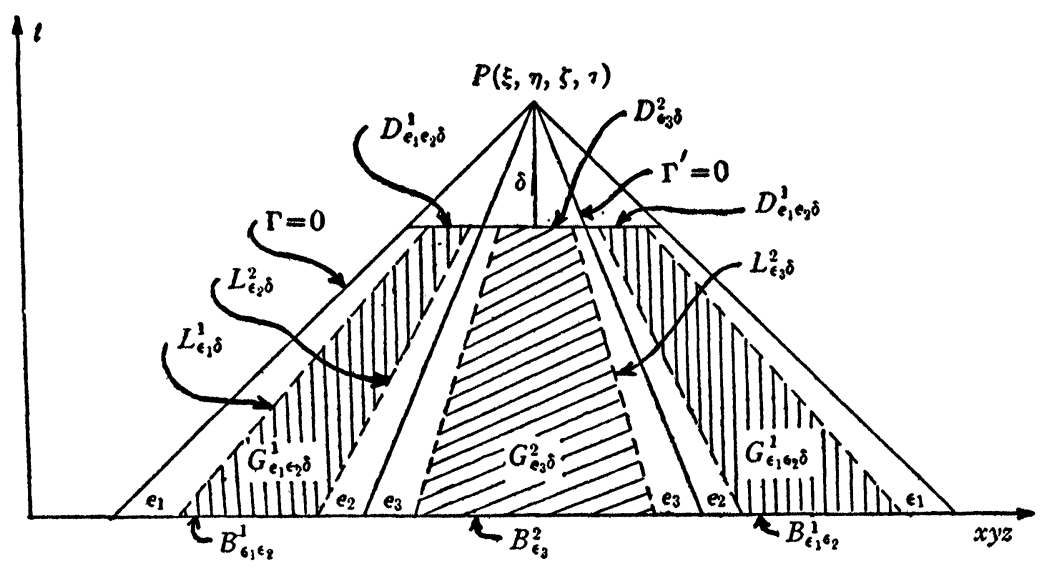

3. Integration over the domain $G_{\mathrm{e}_{1} \epsilon_{2} 8}^{1}$. In $G_{\mathrm{e}_{1038} 8}^{1}$ where $(\tau-t)>r$ and $(\tau-t) / a<r$, the elementary solution is:

$$
V=-\frac{1}{2 r} \log \frac{(\tau-t)-r}{(\tau-t)+r}+\frac{a}{2 r} \log \frac{r-(\tau-t) / a}{r+(\tau-t) / a} .
$$

Since $L[V]=0$, Green's formula (2.3) may be written

$$
\begin{aligned}
-\iiint \int_{G_{\epsilon_{1} \varepsilon_{2} \delta}^{1}} V f d g & +\iiint_{D_{\epsilon_{1} \varepsilon_{2} \delta}^{1}} A d o+\iiint_{L_{\epsilon_{1}}^{1}} A d o \\
& +\iiint_{L_{\epsilon_{2} \delta}^{2}} A d o+\iiint_{B_{\epsilon_{1} \epsilon_{2}}^{1}} A d o=0
\end{aligned}
$$

where

$$
A=V \partial N[u] / \partial s_{2}-u \partial M[V] / \partial s_{1}-N[u] \partial V / \partial s_{2}+M[V] \partial u / \partial s_{1} .
$$

The last integral of (3.1) vanishes due to initial conditions and in order to express the remaining integrals as functions of $\epsilon$ and $\delta$ we introduce new coordinates, $\sigma, \mu ; \alpha, \beta, \gamma$ by the transformation

$$
\begin{array}{ll}
x=\xi+\sigma(1-\mu) \alpha, & y=\eta+\sigma(1-\mu) \beta, \\
z=\zeta+\sigma(1-\mu) \gamma, & t=\tau-\sigma,
\end{array}
$$

where $\alpha, \beta, \gamma$ are parameters on the unit sphere $\alpha^{2}+\beta^{2}+\gamma^{2}=1$. The 
domain $G_{\epsilon_{1} e_{2} \delta}^{1}$ is then determined by the inequalities $\delta \leqq \sigma \leqq \tau$ and $\epsilon_{1} \leqq \mu \leqq 1-1 / a-\epsilon_{2}$, and in the new coordinates:

$$
\begin{gathered}
r=\sigma(1-\mu) ; \quad d x d y d z d t=\sigma^{3}(1-\mu)^{2} d \mu d \sigma d \omega \quad[d \omega=(1 / \gamma) d \alpha d \beta], \\
\Gamma=\sigma^{2} \mu(2-\mu) ; \quad \Gamma^{\prime}=\sigma^{2}\left[1 / a^{2}-(1-\mu)^{2}\right] .
\end{gathered}
$$

We are interested only in the coefficients of $\log \epsilon_{i}$ of each integral which we shall indicate by placing bent bars with the index $i:\langle i\rangle$ in front of the integral sign.

For the first term of (3.1) we obtain the resolution

$$
\begin{aligned}
& -\langle 1,2\rangle \iiint \int_{G_{\epsilon_{1} \epsilon_{2} \delta}^{1}} V f d g \\
& =\langle 1,2\rangle \iiint \int_{G_{\epsilon_{1} \epsilon_{2} \delta}^{1}}\left\{\frac{1}{2 \sigma(1-\mu)} \log \frac{\mu}{2-\mu}\right. \\
& \left.\quad-\frac{a}{2 \sigma(1-\mu)} \log \frac{1-\mu-1 / a}{1-\mu+1 / a}\right\} f \sigma^{8}(1-\mu)^{2} d \mu d \sigma d \omega \\
& =\frac{1}{2} \int_{\delta}^{r} \sigma^{2} d \sigma \iint_{\rho} d \omega\langle 1,2\rangle \int_{\epsilon_{1}}^{1-1 / a-\epsilon_{2}}\{(1-\mu) \log \mu \\
& \quad-a(1-\mu) \log (1-\mu-1 / a)\} f d \mu,
\end{aligned}
$$

where $\rho$ is the surface of the intersection of the conical ring $G_{e_{1} \epsilon_{2} \delta}^{1}$ with the plane $\sigma=\tau-t$. It can be readily seen that upon $\mu$-integration the coefficients of $\log \epsilon_{1}$ and $\log \epsilon_{2}$ will have $\epsilon_{1}$ and $\epsilon_{2}$ respectively as factors and will therefore tend to zero as $\epsilon_{1}$ and $\epsilon_{2}$ approach zero. Therefore,

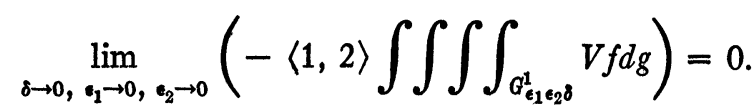

On $D_{\epsilon_{1} \epsilon_{2} 8}^{1}: t_{\nu}=1, x_{\nu}=y_{\nu}=z_{\nu}=0$ and the second term of (3.1) can be written:

$$
\begin{aligned}
\iiint_{D_{\epsilon_{1} e_{2} \delta}^{1}}\left\{a^{2} V \frac{\partial N[u]}{\partial t}-\right. & u \frac{\partial M[V]}{\partial t} \\
& \left.-a^{2} N[u] \frac{\partial V}{\partial t}+M[V] \frac{\partial u}{\partial t}\right\} d x d y d z
\end{aligned}
$$


We consider each of the four integrals of (3.5) separately. The first integral contains $V$ and a resolution similar to that of (3.3) shows that the coefficients of $\log \epsilon_{1}$ and $\log \epsilon_{2}$ tend to zero as $\epsilon_{1}$ and $\epsilon_{2}$ approach zero. Observing that $M[V]=2\left(a^{2}-1\right)(\tau-t) / \Gamma^{2}$, the second integral of $(3.5)$ is:

$$
\begin{aligned}
& -\iiint_{D_{\epsilon_{1} \epsilon_{2} \delta}^{1}} u \frac{\partial M[V]}{\partial t} d x d y d z \\
& \quad=-\left(a^{2}-1\right) \iiint_{D_{\epsilon_{1} \epsilon_{2} \delta}^{1}} u\left\{\frac{8(\tau-t)^{2}}{\Gamma^{3}}-\frac{2}{\Gamma^{2}}\right\} d x d y d z \\
& \quad=-\left(a^{2}-1\right) \iiint_{D_{\epsilon_{1} \varepsilon_{2} \delta}^{1}} u\left\{\frac{8(1-\mu)^{2}}{\sigma \mu^{8}(2-\mu)^{8}}-\frac{2(1-\mu)^{2}}{\sigma \mu^{2}(2-\mu)^{2}}\right\} d \mu d \omega .
\end{aligned}
$$

We note that only terms containing $1 / \mu$ will give terms involving $\log \epsilon_{i}$ after integration. We develop $u$ into a Taylor series in terms of $\bar{u}$, that is, the value of $u$ on the boundary of $D_{e_{1 \varepsilon_{2} \delta} \delta}^{1}$. Since the distance of a point $(\sigma, \mu, \alpha, \beta, \gamma)$ from the corresponding boundary point $\left(\sigma, \epsilon_{1}, \alpha, \beta, \gamma\right)$ is $\sigma\left(1-\epsilon_{1}\right)-\sigma(1-\mu)=\sigma\left(\mu-\epsilon_{1}\right)$, we have $u=\bar{u}-\sigma\left(\mu-\epsilon_{1}\right) u_{v}$ $+\left(\sigma^{2}\left(\mu-\epsilon_{1}\right)^{2} / 2\right) \bar{u}_{\nu \nu}-\cdots=\bar{u}-\sigma \mu \bar{u}_{\nu}+\left(\sigma^{2} \mu^{2} / 2\right) \bar{u}_{\nu \nu}-\cdots+\left(\epsilon_{1}\right)$, where $\left(\epsilon_{1}\right)$ denotes terms which tend to zero with $\epsilon_{1}$ and $\bar{u}_{\nu}=\alpha \partial u / \partial x+\beta \partial u / \partial y$ $+\gamma \partial u / \partial z$. Since the fourth and higher terms in the Taylor development of $u$ contain $\sigma^{8} \mu^{3}$, the difference of $u$ and the first three terms of its development will not give any coefficient of $\log \epsilon_{i}$. For the purpose of determining coefficients of $\log \epsilon_{i}$, we may write therefore

$$
\begin{aligned}
- & \langle 1,2\rangle \iiint_{D_{\epsilon_{1} \epsilon_{2} \delta}^{1}} u \frac{\partial M[V]}{\partial t} d x d y d z \\
= & -\frac{a^{2}-1}{\sigma} \iint_{\rho} u d \omega\langle 1,2\rangle \int_{\epsilon_{1}}^{1-1 / a-\epsilon_{2}}\left\{\frac{8(1-\mu)^{2}}{\mu^{8}(2-\mu)^{3}}-\frac{2(1-\mu)^{2}}{\mu^{2}(2-\mu)^{2}}\right\} d \mu \\
& +\left(a^{2}-1\right) \iint_{\rho} \bar{u}_{\nu} d \omega\langle 1,2\rangle \int_{\epsilon_{1}}^{1-1 / a-\epsilon_{2}}\left\{\frac{8(1-\mu)^{2}}{\mu^{2}(2-\mu)^{3}}-\frac{2(1-\mu)^{2}}{\mu(2-\mu)^{2}}\right\} d \mu \\
& -\frac{\sigma\left(a^{2}-1\right)}{2} \iint_{\rho} \bar{u}_{\nu \nu} d \omega\langle 1,2\rangle \int_{\epsilon_{1}}^{1-1 / a-\iota^{2}}\left\{\frac{8(1-\mu)^{2}}{\mu(2-\mu)^{3}}-\frac{2(1-\mu)^{2}}{(2-\mu)^{2}}\right\} d \mu .
\end{aligned}
$$

The coefficient of $\log \epsilon_{1}$ is zero in the first integral above, 1 in the 
second and $-1 / 2$ in the third. Since the integration does not yield $\log \epsilon_{2}$ we have:

But

$$
\begin{aligned}
\lim _{c_{1} \rightarrow 0, a_{2} \rightarrow 0}(-\langle 1,2\rangle & \left.\iiint_{D_{\epsilon_{1} e_{2} \delta}^{1}} u \frac{\partial M[V]}{\partial t} d x d y d z\right) \\
& =\left(a^{2}-1\right) \iint_{\sigma \leqq \delta} \bar{u}_{\nu} d \omega+\frac{\delta\left(a^{2}-1\right)}{2} \iint_{\sigma=\delta} \bar{u}_{\nu \nu} d \omega .
\end{aligned}
$$

$$
\iint_{\sigma=\delta} \bar{u}_{\nu} d \omega=\frac{1}{\delta^{2}} \iint_{\sigma \leqq \delta} \bar{u}_{\nu} d \Omega=\frac{1}{\delta^{2}} \iiint_{r=\delta} \Delta u d x d y d z,
$$

where the last integral is an infinitesimal of the order $\delta^{8}$ when $\delta$ approaches zero. We find therefore

(3.6) $\lim _{\delta \rightarrow 0} \lim _{\epsilon_{1} \rightarrow 0, \epsilon_{2} \rightarrow 0}\left\{-\langle 1,2\rangle \iiint_{D_{\epsilon_{1} \varepsilon_{2} \delta}^{1}} u \frac{\partial M[V]}{\partial t} d x d y d z\right\}=0$.

The third integral of (3.5) is

$$
\begin{aligned}
-a^{2} & \iiint_{D_{\epsilon_{1} \varepsilon_{2} \delta}^{1}} N[u] \frac{\partial V}{\partial t} d x d y d z \\
& =-a^{2} \iiint_{D_{\epsilon_{1} \varepsilon_{2} \delta}^{1}} N[u]\left\{\frac{1}{\Gamma}-\frac{1}{\Gamma^{\prime}}\right\} d x d y d z \\
& =-\sigma a^{2} \iiint_{D_{\epsilon_{1} \varepsilon_{2} \delta}^{1}} N[u]\left\{\frac{(1-\mu)^{2}}{\mu(2-\mu)}-\frac{(1-\mu)^{2}}{\left[1 / a^{2}-(1-\mu)^{2}\right]}\right\} d \mu d \omega
\end{aligned}
$$

and as we let $\sigma=\delta$ approach zero, this integral vanishes.

The fourth integral of (3.5) is

$$
\begin{aligned}
\langle 1,2\rangle \iiint_{D_{\epsilon_{1} \epsilon_{2} \delta}^{1}} M[ & V] u_{t} d x d y d z \\
& =\left(a^{2}-1\right)\langle 1\rangle \iiint_{D_{\epsilon_{1} \epsilon_{2} \delta}^{1}} \frac{2(\tau-t)}{\Gamma^{2}} u_{t} d x d y d z \\
& =\left(a^{2}-1\right)\langle 1\rangle \iiint_{D_{\epsilon_{1} \epsilon_{2} \delta}^{1}} \frac{2 u_{t}(1-\mu)^{2}}{\mu^{2}(2-\mu)^{2}} d \mu d \omega \\
& =\left(a^{2}-1\right) \iint_{\sigma=\delta} \bar{u}_{t} d \omega\langle 1\rangle \int_{\epsilon_{1}}^{1-1 / a-\epsilon_{2}} \frac{2(1-\mu)^{2}}{\mu^{2}(2-\mu)^{2}} d \mu \\
& =\frac{a^{2}-1}{2} \iint_{\sigma=\delta} \bar{u}_{t} d \omega .
\end{aligned}
$$


Whence

$$
\begin{aligned}
\lim _{\delta \rightarrow 0, \epsilon_{1} \rightarrow 0, \epsilon_{2} \rightarrow 0}\left\{\langle 1\rangle \iiint_{D_{\epsilon_{1} e_{2} \delta}^{1}} M[u] \frac{\partial u}{\partial t} d x d y d z\right\} \\
=2 \pi\left(a^{2}-1\right) u_{t}(\xi, \eta, \zeta, \tau) .
\end{aligned}
$$

On the lateral surface $L_{\epsilon_{1} 8}^{1}, \mu$ is constant and since $M[V]$ and $\partial M[V] / \partial s_{1}$ contain no logarithms, we need only consider

$$
\begin{aligned}
&\langle 1\rangle S_{\epsilon_{1} \delta}^{1}=\langle 1\rangle \iiint_{L_{\epsilon_{1} \delta}^{1}}\left\{V \frac{\partial N[u]}{\partial s_{2}}-N[u] \frac{\partial V}{\partial s_{2}}\right\} d o \\
&=\langle 1\rangle \iiint_{L_{\epsilon_{1} \delta}^{1}}\left\{\left[-\frac{1}{2 \sigma(1-\mu)} \log \frac{\mu}{2-\mu}\right.\right. \\
&\left.\left.\quad+\frac{a}{2 \sigma(1-\mu)} \log \frac{1-\mu-1 / a}{1-\mu+1 / a}\right] \frac{\partial N[u]}{\partial s_{2}}-N[u] \frac{\partial V}{\partial s_{2}}\right\} d o .
\end{aligned}
$$

On the lateral surface $\left(\partial V / \partial s_{2}\right) d o=\left[\left(1-\epsilon_{1}\right) a^{2} V_{t}-\alpha V_{x}-\beta V_{y}-\gamma V_{z}\right]$ $\cdot\left(1-\epsilon_{1}\right)^{2} \sigma^{2} d \sigma d \omega$. Since $V_{t}=1 / \Gamma-1 / \Gamma^{\prime}$, it does not contribute any coefficient of $\log \epsilon_{1}$,

$$
V_{x}=\frac{\alpha}{2 \sigma^{2}(1-\mu)^{2}} \log \frac{\mu}{2-\mu}-\frac{a \alpha}{2 \sigma^{2}(1-\mu)^{2}} \log \frac{1-\mu-1 / a}{1-\mu+1 / a}+\cdots,
$$

where the dots indicate terms which do not contain logarithms. Therefore

$$
\begin{aligned}
\langle 1\rangle \frac{\partial V}{\partial s_{2}} d o & =\langle 1\rangle\left[-\frac{1}{2 \sigma^{2}\left(1-\epsilon_{1}\right)^{2}}\left(\alpha^{2}+\beta^{2}+\gamma^{2}\right)\right]\left(1-\epsilon_{1}\right)^{2} \sigma^{2} d \sigma d \omega \\
& =-d \sigma d \omega / 2 .
\end{aligned}
$$

Thus the coefficient of $\log \epsilon_{1}$ for the lateral surface $L_{\epsilon_{1} \delta}^{1}$ as $\epsilon_{1} \rightarrow 0$ is

$$
\lim _{e_{1} \rightarrow 0}\left(\langle 1\rangle S_{\epsilon_{1} \delta}^{1}\right)=-\frac{1}{2} \iiint_{L_{\delta}^{1}}\left\{\frac{1}{r} \frac{\partial N[u]}{\partial s_{2}}-N[u] \frac{\partial(1 / r)}{\partial s_{2}}\right\} d o .
$$

In the limit $\delta \rightarrow 0$, we obtain

$$
\begin{aligned}
& \lim _{\delta \rightarrow 0, c_{1} \rightarrow 0}\left(\langle 1\rangle S_{\epsilon_{1} \delta}^{1}\right) \\
& \quad=-\frac{1}{2} \iiint_{L^{1}}\left\{\frac{1}{r} \frac{\partial N[u]}{\partial s_{2}}-N[u] \frac{\partial(1 / r)}{\partial s_{2}}\right\} d o .^{2}
\end{aligned}
$$

${ }^{2}$ Since $\partial(1 / r) / \partial s_{2}$ is not defined at the point $P(\xi, \eta, \zeta, \tau)$, this integral is to be taken in the limiting sense. 
Similarly on the lateral surface $L_{\epsilon_{2} \delta}^{2}$ we need only consider

$$
\langle 2\rangle S_{\mathrm{e}_{2} \delta}^{2}=\langle 2\rangle \iiint_{L_{\mathrm{c}_{2} \delta}^{2}}\left\{V \frac{\partial N[u]}{\partial s_{2}}-N[u] \frac{\partial V}{\partial s_{2}}\right\} d o .
$$

We have $\langle 2\rangle V=a / 2 r ;\langle 2\rangle \partial V / \partial s_{2}=(a / 2) \partial(1 / r) / \partial s_{2}$. Therefore the coefficient of $\log \epsilon_{2}$ for the lateral surface $L_{\epsilon_{2} \delta}^{2}$ is, when $\epsilon_{2} \rightarrow 0$,

$$
\lim _{\epsilon_{2} \rightarrow 0}\left(\langle 2\rangle S_{\epsilon_{2} \delta}^{2}\right)=\frac{a}{2} \iiint_{L_{\delta}^{2}}\left\{\frac{1}{r} \frac{\partial N[u]}{\partial s_{2}}-N[u] \frac{\partial(1 / r)}{\partial s_{2}}\right\} d o .
$$

In the limit $\delta \rightarrow 0$, we obtain:

(3.9) $\lim _{\delta \rightarrow 0} \lim _{e_{2} \rightarrow 0}\left(\langle 2\rangle S_{\epsilon_{2} \delta}^{2}\right)=\frac{a}{2} \iiint_{L^{2}}\left\{\frac{1}{r} \frac{\partial N[u]}{\partial s_{2}}-N[u] \frac{\partial(1 / r)}{\partial s_{2}}\right\} d o$.

Summarizing the results obtained by the use of the first form of Green's formula (2.3), we have the equation

$$
\begin{array}{r}
{\left[2 \pi\left(a^{2}-1\right) u_{t}(P)-\frac{1}{2} \iiint_{L^{1}}\left\{\frac{1}{r} \frac{\partial N[u]}{\partial s_{2}}-N[u] \frac{\partial(1 / r)}{\partial s_{2}}\right\} d o\right] \log \epsilon_{1}} \\
+\left[\frac{a}{2} \iiint_{L^{2}}\left\{\frac{1}{r} \frac{\partial N[u]}{\partial s_{2}}-N[u] \frac{\partial(1 / r)}{\partial s_{2}}\right\} d o\right] \log \epsilon_{2}=0 .
\end{array}
$$

Since $\epsilon_{1}$ and $\epsilon_{2}$ are independent, the coefficients of $\log \epsilon_{1}$ and $\log \epsilon_{2}$ must vanish, which gives the following relations:

$$
\begin{aligned}
& 2 \pi\left(a^{2}-1\right) u_{t}(P)=\frac{1}{2} \iiint_{L^{\prime}}\left\{\frac{1}{r} \frac{\partial N[u]}{\partial s_{2}}-N[u] \frac{\partial(1 / r)}{\partial s_{2}}\right\} d o \\
& \quad \frac{a}{2} \iiint_{L^{2}}\left\{\frac{1}{r} \frac{\partial N[u]}{\partial s_{2}}-N[u] \frac{\partial(1 / r)}{\partial s_{2}}\right\} d o=0 .
\end{aligned}
$$

4. Application of the second form of Green's formula and integration over the domain $G_{e 88}^{2}$. In a manner quite analogous to that in the preceding section we perform the integration of the second form of Green's formula (2.4) over the domains $G_{\epsilon_{1} \epsilon_{2} \delta}^{1}$ and $G_{\epsilon_{8} \delta}^{2}$ and obtain the following additional relations:

$$
\begin{aligned}
& \frac{1}{2} \iiint_{L^{1}}\left\{\frac{1}{r} \frac{\partial M[u]}{\partial s_{1}}-M[u] \frac{\partial(1 / r)}{\partial s_{1}}\right\} d o=0 \\
& 2 \pi a\left(a^{2}-1\right) u_{t}(P) \\
& \quad=-\frac{a}{2} \iiint_{L^{2}}\left\{\frac{1}{r} \frac{\partial M[u]}{\partial s_{1}}-M[u] \frac{\partial(1 / r)}{\partial s_{1}}\right\} d o .
\end{aligned}
$$


Subtracting (4.2) from (3.10) we obtain an expression for $u_{t}$ :

$$
\begin{aligned}
2 \pi(1-a)\left(a^{2}-1\right) u_{t}(P) & \\
= & \frac{1}{2} \iiint_{L^{1}}\left\{\frac{1}{r} \frac{\partial N[u]}{\partial s_{2}}-N[u] \frac{\partial(1 / r)}{\partial s_{2}}\right\} d o \\
& +\frac{a}{2} \iiint_{L^{2}}\left\{\frac{1}{r} \frac{\partial M[u]}{\partial s_{1}}-M[u] \frac{\partial(1 / r)}{\partial s_{1}}\right\} d o .
\end{aligned}
$$

5. Transformation of lateral surface integrals into volume integrals. Let us consider the self-adjoint expression $M[u]=a^{2} \partial^{2} u / \partial t^{2}-\Delta u$. Green's formula for this expression of the second order is

$$
\begin{aligned}
\iiint \int_{G}\{V M[u]-u M[V]\} d g \\
=\iiint_{S}\left\{V \frac{\partial u}{\partial s_{2}}-u \frac{\partial V}{\partial s_{2}}\right\} d o,
\end{aligned}
$$

where $S$ is the three-dimensional boundary of $G$. If we put $V=1 / r$ and $u=N[u]$, we cannot apply (5.1) over the interior of the surface $\Gamma=0$ because $V$ has a singularity on the line $r=0$. To overcome this difficulty we again truncate the cone $\Gamma=0$ by the plane $t=\tau-\delta$ and remove the line $r=0$ by cutting out a small cone $\epsilon^{2}(\tau-t)^{2}-r^{2}=0$. Over this new domain, which we denote by $G_{\epsilon \delta}^{1}$, we apply formula (5.1):

$$
\begin{aligned}
& \iiint \int_{G_{\epsilon \delta}^{1}}\left\{\frac{1}{r} M[N[u]]\right.\left.-N[u] M\left[\frac{1}{r}\right]\right\} d g \\
&=\iiint_{S_{\epsilon \delta}^{1}}\left\{\frac{1}{r} \frac{\partial N[u]}{\partial s_{2}}-N[u] \frac{\partial(1 / r)}{\partial s_{2}}\right\} d o .
\end{aligned}
$$

Since $M[1 / r]=0$ and, by (2.1), $M[N[u]]=f(x, y, z, t)$, we have

$$
\begin{aligned}
\iiint \int_{a_{\delta}} \frac{1}{r} f d g & =\iiint_{L_{\delta}^{1}}\left\{\frac{1}{r} \frac{\partial N[u]}{\partial s_{2}}-N[u] \frac{\partial(1 / r)}{\partial s_{2}}\right\} d o \\
& +\iiint_{D_{\epsilon \delta}^{1}}\left\{\frac{a^{2}}{r} \frac{\partial N[u]}{\partial t}-a^{2} N[u] \frac{\partial(1 / r)}{\partial t}\right\} d x d y d z \\
& +\iiint_{L_{\epsilon \delta}}\left\{\frac{1}{r} \frac{\partial N[u]}{\partial s_{2}}-N[u] \frac{\partial(1 / r)}{\partial s_{2}}\right\} d o \\
& -\iiint_{B_{\epsilon}^{1}}\left\{\frac{a^{2}}{r} \frac{\partial N[u]}{\partial t}-a^{2} N[u] \frac{\partial(1 / r)}{\partial t}\right\} d x d y d z .
\end{aligned}
$$


The last integral of (5.2) vanishes because of initial conditions. Since

$$
\begin{aligned}
\iiint_{D_{\epsilon \delta}^{1}} & \left\{\frac{a^{2}}{r} \frac{\partial N[u]}{\partial t}-a^{2} N[u] \frac{\partial(1 / r)}{\partial t}\right\} d x d y d z \\
& =\sigma^{3} \iiint_{D_{\epsilon \delta}^{1}}\left\{\frac{a^{2}}{r} \frac{\partial N[u]}{\partial t}-a^{2} N[u] \frac{\partial(1 / r)}{\partial t}\right\}(1-\mu)^{2} d \mu d \omega,
\end{aligned}
$$

the integral over $D_{e \delta}^{1}$ vanishes in the limit $\epsilon \rightarrow 0, \sigma=\delta \rightarrow 0$. In order to find the limiting value of the integral over $L_{\ell \delta}$, we make the following transformation of the coordinates:

$$
x=\xi+\epsilon \sigma \alpha, \quad y=\eta+\epsilon \sigma \beta, \quad z=\zeta+\epsilon \sigma \gamma, \quad t=\tau-\sigma .
$$

In the new coordinates:

$$
r=\epsilon \sigma, \quad d o=\epsilon^{2} \sigma^{2}\left(1+\epsilon^{2}\right)^{1 / 2} d \sigma d \omega, \quad \partial(1 / r) / \partial s_{2}=-1 / r^{2}\left(1+\epsilon^{2}\right)^{1 / 2},
$$

and we obtain:

$$
\begin{aligned}
& \iiint_{L_{\epsilon \delta}}\left\{\frac{1}{r} \frac{\partial N[u]}{\partial s_{2}}-N[u] \frac{\partial(1 / r)}{\partial s_{2}}\right\} d o \\
& =\iiint_{L_{\epsilon \delta}}\left\{\frac{1}{\epsilon \sigma} \frac{\partial N[u]}{\partial s_{2}}+N[u] \frac{1}{\epsilon^{2} \sigma^{2}\left(1+\epsilon^{2}\right)^{1 / 2}}\right\} \epsilon^{2} \sigma^{2}\left(1+\epsilon^{2}\right)^{1 / 2} d \sigma d \omega .
\end{aligned}
$$

In the limit $\epsilon \rightarrow 0, \delta \rightarrow 0$ we obtain

$$
\lim _{\alpha \rightarrow 0, \delta \rightarrow 0}\left\{\iint_{r=\delta \epsilon} d \omega \int_{\delta}^{\tau} N[u] d \sigma\right\}=-4 \pi \int_{0}^{\tau} N[u] d t .
$$

Therefore as we let $\epsilon$ and $\delta$ approach zero, (5.2) gives

$$
\begin{aligned}
\iiint \int_{G^{1}} \frac{1}{r} f d g= & \iiint_{L^{1}}\left\{\frac{1}{r} \frac{\partial N[u]}{\partial s_{2}}-N[u] \frac{\partial(1 / r)}{\partial s_{2}}\right\} d o \\
& -4 \pi \int_{0}^{r} N[u] d t .
\end{aligned}
$$

Interchanging $N[u]$ with $M[u]$ we obtain a similar expression, but, since by (4.1) the integral over $L^{1}$ vanishes, we have the relation

$$
\iiint \int_{G^{1}} \frac{1}{r} f d g=-4 \pi \int_{0}^{r} M[u] d t .
$$

The same procedure applied to the domain $G^{2}$ consisting of the interior of the surface $\Gamma^{\prime}=0$ yields the following relations: 


$$
\begin{aligned}
\iiint \int_{G^{2}} \frac{1}{r} f d g= & \iiint_{L^{2}}\left\{\frac{1}{r} \frac{\partial M[u]}{\partial s_{1}}-M[u] \frac{\partial(1 / r)}{\partial s_{1}}\right\} d o \\
& -4 \pi \int_{0}^{r} M[u] d t,
\end{aligned}
$$

$$
\iiint \int_{G^{2}} \frac{1}{r} f d g=-4 \pi \int_{0}^{r} N[u] d t .
$$

6. Solution of the initial value problem. The relations of the preceding section enable us to obtain the solution of the initial value problem (2.1). Combining (5.3) and (5.6) we obtain

$$
\begin{aligned}
\iiint_{L^{1}}\left\{\frac{1}{r} \frac{\partial N[u]}{\partial s_{2}}\right. & \left.-N[u] \frac{\partial(1 / r)}{\partial s_{2}}\right\} d o \\
& =\iiint \int_{G^{1}} \frac{1}{r} f d g-\iiint \int_{G^{2}} \frac{1}{r} f d g,
\end{aligned}
$$

and combining (5.4) and (5.5) gives the relation

$$
\begin{aligned}
\iiint_{L^{2}}\left\{\frac{1}{r} \frac{\partial M[u]}{\partial s_{1}}\right. & \left.-M[u] \frac{\partial(1 / r)}{\partial s_{1}}\right\} d o \\
& =\iiint \int_{G^{2}} \frac{1}{r} f d g-\iiint \int_{G^{1}} \frac{1}{r} f d g .
\end{aligned}
$$

Substitution of the expressions (6.1) and (6.2) for the lateral surface integrals in (4.3) gives

$$
\begin{aligned}
4 \pi(1-a)\left(a^{2}-1\right) u_{t}(P)=(1-a) & \iiint \int_{G^{1}} \frac{1}{r} f d g \\
& -(1-a) \iiint \int_{G^{2}} \frac{1}{r} f d g
\end{aligned}
$$

or

(6.3) $4 \pi\left(a^{2}-1\right) u_{t}(P)=\iiint \int_{G^{1}} \frac{1}{r} f d g-\iiint \int_{G^{2}} \frac{1}{r} f d g$.

Therefore the solution of the initial value problem (2.1) is

$$
\begin{aligned}
4 \pi\left(a^{2}-1\right) u(\xi, \eta, \zeta, \tau)= & \iiint \int_{a^{1}} \frac{\tau-t-r}{r} f d g \\
& -\iiint \int_{G^{2}} \frac{\tau-t-a r}{r} f d g .
\end{aligned}
$$


In order to show that the right-hand side of (6.4) has the required derivatives we apply thereto transformation (3.2) and obtain

$$
\begin{aligned}
& \iiint \int_{G^{1}} f \sigma^{3} \mu(1-\mu) d \sigma d \mu d \omega \\
&-\iiint \int_{G^{2}} f \sigma^{3}[1-a(1-\mu)](1-\mu) d \sigma d \mu d \omega .
\end{aligned}
$$

Because of our assumptions concerning the function $f$ both integrands are regular and our proof is complete.

7. The limiting case $a=1$. In order to determine what happens when $a=1$, we observe that the solution (6.4) for $a>1$ can be written in the form

$$
\begin{aligned}
4 \pi\left(a^{2}-1\right) u(P)= & \int_{0}^{\tau} d t \iiint_{r \leqq r-t} \frac{\tau-t-r}{r} f d x d y d z \\
& -\int_{0}^{r} d t \iiint_{r \leqq(\tau-t) / a} \frac{\tau-t-a r}{r} f d x d y d z \\
= & F(1)-F(a) .
\end{aligned}
$$

Therefore $4 \pi(a+1) u(P)=-[F(1)-F(a)] /(1-a)$ and in the limit $a=1,8 \pi u(P)=-F^{\prime}(1)$. But differentiation of $F(a)$ with respect to $a$ gives

whence

$$
F^{\prime}(a)=-\int_{0}^{\tau} d t \iiint_{r \leqq(\tau-t) / a} f d x d y d z,
$$

$$
F^{\prime}(1)=-\int_{0}^{\tau} d t \iiint_{r \geqq \tau-t} f d x d y d z
$$

and the solution of the iterated wave equation is

$$
8 \pi u(P)=\iiint \int_{G^{1}} f d x d y d z d t .
$$

8. Nonhomogeneous initial conditions. We consider now equation (2.1) with the following nonhomogeneous initial conditions:

$$
\begin{aligned}
& u(x, y, z, 0)=\phi_{0}(x, y, z), \quad u_{t}(x, y, z, 0)=\phi_{1}(x, y, z), \\
& u_{t t}(x, y, z, 0)=\phi_{2}(x, y, z), \quad u_{t t t}(x, y, z, 0)=\phi_{3}(x, y, z) .
\end{aligned}
$$

The introduction of the initial conditions (8.1) does not present anything new with regard to our method and we content ourselves in writing the solution which satisfies such conditions: 


$$
\begin{aligned}
4 \pi\left(a^{2}-1\right) u(\xi, \eta, \zeta, \tau) & =\iiint \int_{a^{1}} \frac{\tau-t-r}{r} f d x d y d z d t \\
= & -\iiint \int_{G^{2}} \frac{\tau-t-a r}{r} f d x d y d z d t \\
& +\iiint_{r \leqq \tau} \frac{1}{r}\left\{(\tau-r)\left[a^{2} \phi_{3}-\left(a^{2}+1\right) \Delta \phi_{1}\right]\right. \\
& -\iiint_{r \leqq \tau / a} \frac{1}{r}\left\{(\tau-a r)\left[a^{2} \phi_{3}-\left(a^{2}+1\right) \Delta \phi_{1}\right]\right. \\
& +\frac{\left.\left.a^{2} \phi_{2}-\left(a^{2}+1\right) \Delta \phi_{0}\right]\right\} d x d y d z}{\tau} \iint_{r \leqq r} \Delta \phi_{0} d x d y d z-\frac{a}{\tau} \iiint_{r \leqq \tau / a} \Delta \phi_{0} d x d y d z \\
& +\frac{a^{2}}{\tau} \iint_{\tau=\tau}\left(\phi_{1}+\frac{1}{\tau} \phi_{0}\right) d S \\
& -\frac{a^{2}}{\tau} \iint_{r=\tau / a}\left(\phi_{1}+\frac{1}{\tau} \phi_{0}\right) d S .
\end{aligned}
$$

In the limiting case $a=1$, the above result reduces to

$$
\begin{aligned}
8 \pi u(\xi, \eta, \zeta, \tau) & \\
= & \iiint \int_{G^{1}} f d g+\iiint_{r \leqq \tau}\left(\phi_{3}-\Delta \phi_{1}+\frac{2}{\tau} \Delta \phi_{0}\right) d x d y d z \\
& +\iint_{r=\tau}\left(\phi_{2}+\frac{2}{\tau} \phi_{1}+\frac{2}{\tau^{2}} \phi_{0}-\Delta \phi_{0}\right) d S
\end{aligned}
$$

\section{BIBLIOGRAPHY}

1. J. Hadamard, Lectures on Cauchy's problem in linear partial differential equations, Yale University Press, 1923.

2. B. Riemann, On the propagation of aerial waves, Göttingen Abhandlungen vol. 8 (1860).

3. K. Friedrichs, Die Verallgemeinerung der Riemannschen Methode auf eine beliebige Anzahl von Dimensionen, Nachr. Ges. Wiss. Göttingen, 1927, pp. 172-177.

4. R. Courant and D. Hilbert, Methoden der Mathematischen Physik, vol. II, pp. 430-448.

Brooklyn College and New York University 